\title{
BMJ Open Risk factors for progression of carotid intima-media thickness in patients with systemic lupus erythematosus: protocol for an observational cohort study in China
}

\author{
Haiyu Pang, ${ }^{1}$ Yicong Ye, ${ }^{2}$ Faming Ding, ${ }^{2}$ Mengtao $L i,{ }^{3}$ Xinglin Yang, ${ }^{2}$ Xufei Yang, ${ }^{2}$ \\ Qian Wang, ${ }^{3}$ Dong Xu, ${ }^{3}$ Yunyun Fei, ${ }^{3}$ Lin Kang, ${ }^{4}$ Xiaofeng Zeng, ${ }^{3}$ Shuyang Zhang ${ }^{2}$
}

To cite: Pang $\mathrm{H}, \mathrm{Ye} \mathrm{Y}$, Ding $\mathrm{F}$, et al. Risk factors for progression of carotid intimamedia thickness in patients with systemic lupus erythematosus: protocol for an observational cohort study in China. BMJ Open 2019;9:e030721. doi:10.1136/ bmjopen-2019-030721

- Prepublication history for this paper is available online. To view these files please visit the journal online (http://dx.doi org/10.1136/bmjopen-2019030721).

Received 29 March 2019 Revised 17 July 2019 Accepted 18 July 2019

A Check for updates

(C) Author(s) (or their employer(s)) 2019. Re-use permitted under CC BY-NC. No commercial re-use. See rights and permissions. Published by BMJ.

For numbered affiliations see end of article.

Correspondence to Dr Shuyang Zhang; shuyangzhang103@nrdrs.org

Dr Xiaofeng Zeng; pumchzx@@163.com

\section{ABSTRACT}

Introduction Accelerated atherosclerosis is a major complication of systemic lupus erythematosus (SLE), and it leads to increased cardiovascular morbidity and mortality in patients with SLE. This study aimed to investigate the natural progression of carotid intima-media thickness (CIMT), and to examine the risk factors for progression of CIMT and atherosclerotic plaques based on a Chinese SLE cohort.

Methods and analysis Participants were continuously enrolled as outpatients of the Department of Rheumatology in Peking Union Medical College Hospital (PUMCH) from October 2013 to December 2016. Inclusion criteria were as follows: (1) age $\geq 18$ years, (2) fulfilment of clinical classification criteria of SLE and (3) provision of signed written informed consent. Patients with clinically overt coronary artery disease, a history of cardiovascular disease (previous stroke, heart failure, myocardial infarction, angina or symptomatic peripheral artery disease) and malignancy, and pregnant/lactating women were excluded. The primary outcome is progression of CIMT from baseline. A total of 440 patients with SLE will be enrolled. Participants will receive follow-up surveys $\sim 5$ years after their baseline visit. A standard structural survey form, including demographic data, medical history, clinical and laboratory assessments and CIMT measurement, is planned for data collection at baseline and follow-up. The risk prediction model for progression of CIMT will be created by using a mixed effect model.

Ethics and dissemination The study protocol was approved by the institutional review board of PUMCH (S-599). Informed consent was obtained from all participants according to the Declaration of Helsinki on Biomedical Research Involving Human Studies. All data will be managed confidentially according to guidelines and legislation. Dissemination will include publication of scientific papers and/or presentations of the study findings at international conferences.

\section{INTRODUCTION}

Accelerated atherosclerosis is a major complication of systemic lupus erythematosus (SLE), and it leads to increased cardiovascular
Strengths and limitations of this study

- This is the first prospective cohort to monitor carotid intima-media thickness (CIMT) among patients with systemic lupus erythematosus (SLE) in China.

- The risk prediction model for progression of CIMT in SLE will be created by using a mixed effect model.

- The sample size is relatively large, and we compensated for individual factors that may affect progression of atherosclerosis.

- This was not an inception cohort, and $~ 40 \%$ of patients were in their first year since SLE was diagnosed at baseline.

- Most participants are from the north of China because of the geographical position of the Peking Union Medical College Hospital, and our results might only be representative for patients with SLE in North China.

morbidity and mortality in patients with SLE. ${ }^{1-4}$ Prevention and intervention of premature atherosclerosis is beneficial for prognosis and survival of patients with SLE.

Although the exact pathogenesis of accelerated atherosclerosis in SLE remains poorly defined, endothelial dysfunction and dysregulation of immune responses are the areas of greatest concern by researchers. ${ }^{5}$ SLE may affect the integrity and repair mechanisms of endothelial cells through direct binding of antibodies to endothelial cells or deposition of circulating immune complexes. This then results in endothelial damage that promotes atherogenesis. ${ }^{6}$ Accelerated atherosclerosis in SLE may also be related to the presence of antiphospholipid antibodies, which increase the risk of thrombosis in SLE. ${ }^{7-9}$

Traditional Framingham risk factors, including age, sex, hyperlipidemia, smoking, hypertension and $\mathrm{C}$ reactive protein, partly 
explain, but do not account entirely, for the increased incidence of premature atherosclerosis in patients with SLE. ${ }^{10-13}$ Recent studies have shown that factors related to SLE, medication, psychological stress and novel non-traditional factors, such as inflammation, are likely to contribute to development of premature atherosclerosis. ${ }^{14-21}$ Most studies have established an association between risk factors and accelerated atherosclerosis on the basis of frequency of myocardial infarction, stroke or cardiovascular deaths among patients with SLE. ${ }^{22} 23$ However, evidence based on the process of quantitative monitoring of atherosclerosis is still lacking.

Several studies have demonstrated the usefulness of carotid intima-media thickness (CIMT) in predicting future vascular events. ${ }^{24}{ }^{25}$ Therefore, CIMT could be used as a quantitative clinical surrogate endpoint for the risk of accelerated atherosclerosis in patients with SLE. However, most results using CIMT as a surrogate endpoint have come from retrospective studies or cross-sectional studies. Little evidence has been reported longitudinally on the pace and risk factors for progression of CIMT in SLE.

Therefore, the primary aim of this study was to investigate the natural progress of CIMT in 5 years, and examine the risk factors for progression of CIMT and atherosclerotic plaques based on a Chinese SLE cohort. The secondary aims of the study were to investigate progression of brachial-ankle pulse wave velocity (baPWV), and to examine the risk factors for increasing baPWV in patients with SLE. Our findings may provide a reference for prevention and intervention strategies for premature atherosclerosis in patients with SLE.

\section{METHODS AND ANALYSIS \\ Main hypotheses}

From a population point of view, all patients with SLE have a risk of developing atherosclerosis. Therefore, as a whole, patients with SLE are a population at risk for atherosclerosis. Each individual in this high-risk

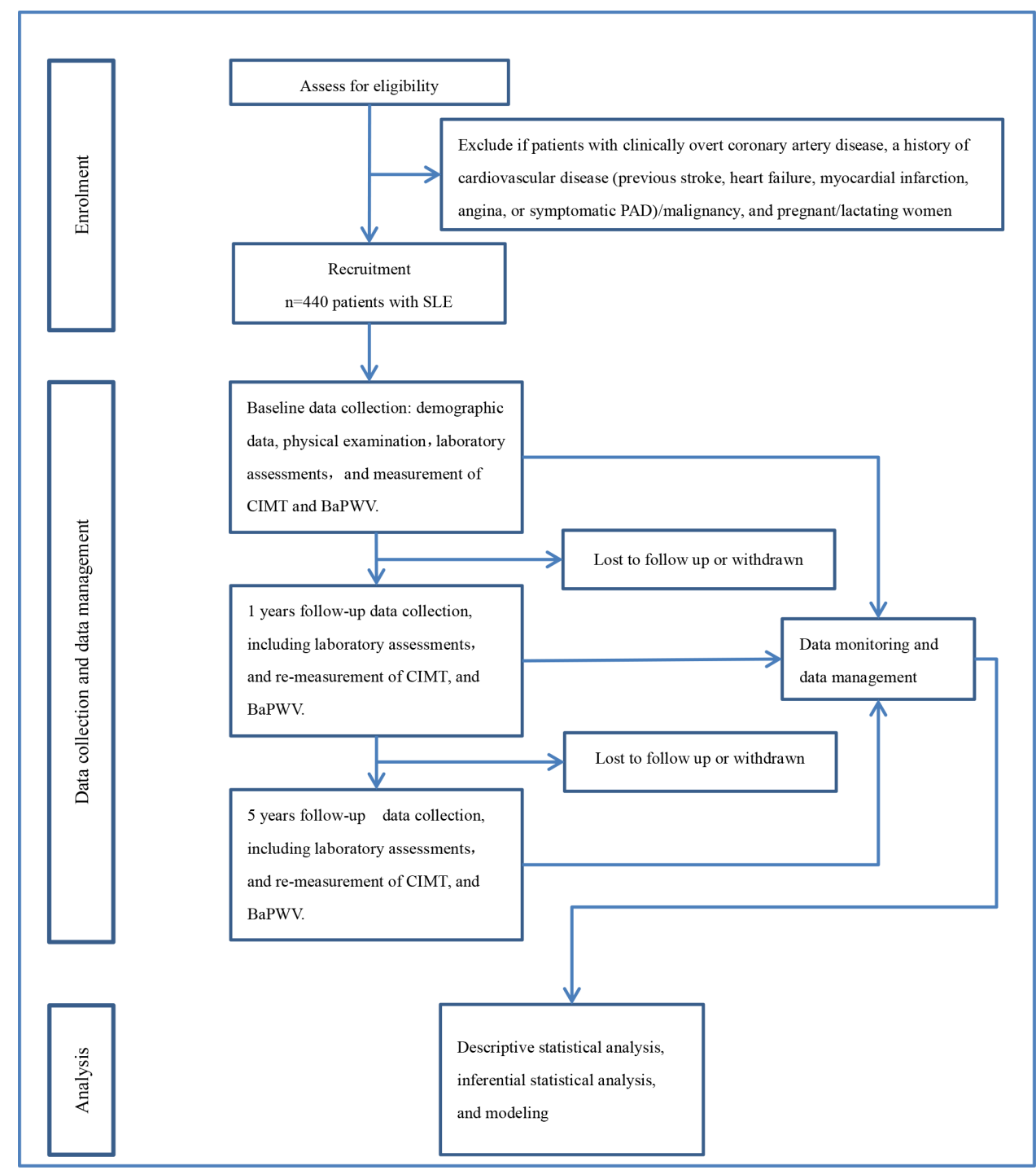

Figure 1 Study procedure and time points of data collection. baPWV, brachial-ankle pulse wave velocity; CIMT, carotid intimamedia thickness; SLE, systemiclupus erythematosus. 
population has different personal risk factors (eg, age, sex, disease duration). This means that their corresponding levels of risk for developing atherosclerosis are not the same. Under such an assumption, we conducted a cohort study to investigate the change in CIMT, examine the risk factors for progression of CIMT and create a risk prediction model on the basis of a Chinese SLE cohort.

\section{Study design and timeline}

This study was a prospective cohort in design and was initiated in October 2013. The study procedure is shown in figure 1. The plan for two stages of data collections is as follows.

\section{Baseline}

The overall design and pilot study were conducted from May 2013 to September 2013. We recruited participants and collected baseline data from October 2013 to December 2016. These data comprised demographic information, medical history, clinical and laboratory assessments, as well as baseline CIMT and baPWV, in patients with SLE.

\section{Five-year follow-up}

We will conduct clinical and laboratory assessments, and re-evaluate CIMT and baPWV from October 2018 to December 2021.

\section{Planned substudies}

The project includes two planned substudies. In substudy 1 , we will evaluate differences in arterial stiffness in different age groups among patients with SLE. In substudy 2 , we will examine the relationship between the interarm blood pressure difference and peripheral artery disease, which will be diagnosed with the ankle-brachial index in patients with SLE.

\section{Organisational structure}

The Central Project Group is at the top of the organisational structure, and is responsible for designing, supervising the entire process, checking data quality, statistical analysis, proposing interim seminars and project conferences and disseminating all project materials and protocols (figure 2). The following layers of the process comprise functional teams, which are further divided into two groups as follows: (1) clinical doctor team of cardiology, rheumatology and ultrasound; and (2) supporting teams that are responsible for constructing an information system, maintenance and updates.

\section{Study population}

The target population was patients with the clinical diagnosis of SLE. Study participants were recruited through a nationwide registry system created by the Chinese SLE Treatment and Research group. ${ }^{26-31}$ All patients were continuously enrolled as outpatients of the Department of Rheumatology in Peking Union Medical College Hospital (PUMCH), which is a renowned hospital in China.

Inclusion criteria for the study were as follows: (1) age $\geq 18$ years, (2) fulfilment of clinical classification criteria of SLE (four or more 1997 American College of Rheumatology classification criteria) ${ }^{32}$ and (3) provision of signed written informed consent. Patients with clinically overt coronary artery disease, a history of cardiovascular disease (previous stroke, heart failure, myocardial infarction, angina, or symptomatic peripheral artery disease) and malignancy, and pregnant/lactating women were excluded.

\section{Outcome measures and data collection Major study outcomes}

The primary outcome is the quantitative change in CIMT from baseline. The secondary outcome is the quantitative change in baPWV from baseline.

\section{Clinical data collection}

A standard structural survey form that included demographic data, medical history and clinical and laboratory assessments was used for data collection. All patients with SLE underwent a complete medical history survey and physical examination, according to the study protocol. Traditional risk factors of coronary artery disease (eg, age, family history, hypertension, diabetes mellitus and specific therapy, cholesterol, body mass index, current smoking

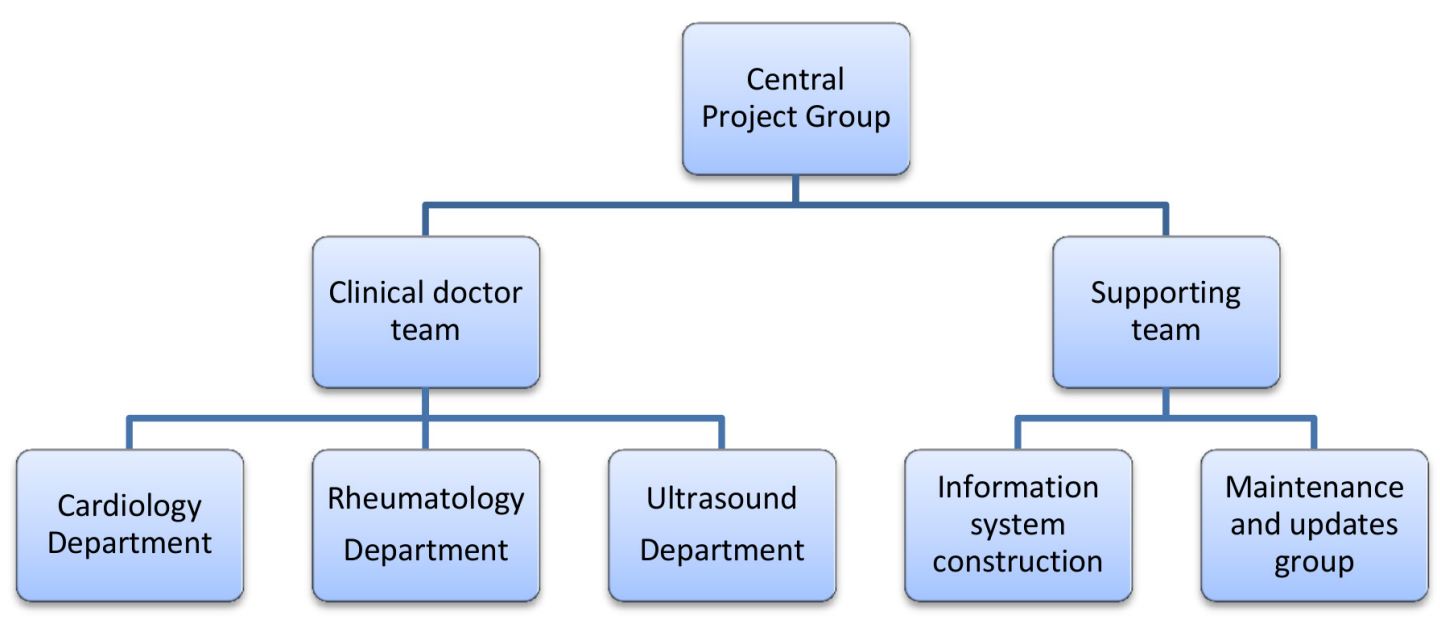

Figure 2 Organisational structure of the project. 
status and smoking history) and lupus-specific factors (eg, disease activity, duration of the disease, organ involvement, cumulative dose of steroids, current and initial dose of prednisone and use of antimalarial and immunosuppressive medications) that may affect development of coronary artery disease were determined by interviewing patients. Disease activity and damage were assessed using the SLE disease activity index and the Systemic Lupus International Collaborating Clinics Damage Index, respectively. ${ }^{18}$

\section{CIMT measurement}

An ultrasound scan of CIMT was performed in all participants using a standard protocol at baseline and at the follow-up visit. Ultrasound was performed by using a Philips IU22 ultrasound system with a 9-3 and 12-5 MHz linear array transducer. The patient was placed in the supine position for ultrasonographic examination of the carotid artery. The far wall CIMT was measured on the far wall of the common carotid arteries $(1 \mathrm{~cm})$, bifurcation $(1 \mathrm{~cm})$ and distal internal carotid artery $(1 \mathrm{~cm})$ at the bifurcation, with a total length of $3 \mathrm{~cm}$ of the proximal carotid artery (anterior wall) and distal carotid artery (posterior wall). The ultrasound scan was performed by an experienced sonographer and interpreted by a single highly experienced cardiologist who was blinded to the clinical characteristics of the participants.

According to sonographic criteria, a normal CIMT is $<0.9 \mathrm{~mm}$, an increased CIMT $(>0.9 \mathrm{~mm})$ is considered indicative of a thickened intima and a CIMT $>1.3 \mathrm{~mm}$ indicates an atherosclerotic plaque. ${ }^{334}$

\section{Pulse wave velocity measurement}

Blood pressure and baPWV were measured using a noninvasive vascular screening device (BP-203RPEIII VP-1000; Omron Corp, Kyoto, Japan). This device simultaneously records an ECG, phonocardiogram and pulse volume, and calculates the time delay of the pulse to obtain pulse wave transmission time.

\section{Laboratory assessments}

Blood tests included fasting plasma glucose levels, glycated haemoglobin levels, the erythrocyte sedimentation rate, complement 3 (C3) levels, C4 levels and routine biochemical parameters, including urea, creatinine, albumin, triglyceride, total cholesterol, low-density lipoprotein cholesterol and high-density lipoprotein cholesterol levels. Biochemical parameters were measured by routine laboratory methods. Data on antinuclear antibody, anti-doublestranded DNA and anticardiolipin antibodies to IgM and IgG, as determined by an ELISA method within 3 months before enrolment, were extracted from the patients' medical records.

\section{Follow-up}

All participants will be followed longitudinally for 1 and 5 years after enrolment at the Department of Rheumatology, PUMCH. Clinical assessments, laboratory assessments, and CIMT and baPWV measurements will be repeated at the follow-up visit. For a change in treatment (especially steroids), we will obtain the change in medication type and dosage by the patient's medical record, as well as by visiting the patient.

\section{Quality assurance}

All data collectors were trained according to unified standards of data extraction and form completion. Survey forms were completed by doctoral candidates of internal medicine and rechecked by clinicians to ensure data accuracy and logicality.

All data were uploaded to the registry system data centre by the study data manager. The central project group will perform random checks of data against logic and the original paper forms. An item error rate of $\geq 5.0 \%$ will result in rejection and repeated entry. Individual questions and errors can be resolved by discussing them with the clinical team. In terms of data security, authority for raw data scanning will be restricted to the central project group level and only specified project personnel have authority to access the entire database.

\section{Data analysis plan}

Data processing and statistical analyses were performed using SAS V.9.3. Analysis of baseline data was conducted by age group to describe the disease status and basic socioeconomic characteristics of the patients with SLE. Normally distributed continuous data are expressed as mean \pm SD and non-normally distributed continuous data are expressed as the median (25th percentile, 75th percentile). A test for normality was performed by the Shapiro-Wilk test. Categorical variables are expressed as a constituent ratio or rate. The $\chi^{2}$ or Fisher's exact test was performed to test comparability between patients in different subgroups. Baseline data were analysed cross-sectionally to determine the relationship between risk factors and CIMT/baPWV by using appropriate statistical methods (eg, linear regression analysis, analysis of covariance, logistic regression analysis), with adjustment for potential covariates, such as age and disease duration.

Follow-up data will be analysed longitudinally to calculate the change in CIMT/baPWV. The linear mixed effect model will be used to identify risk factors (traditional risk factors and lupus-specific factors) for progression of CTMT/baPWV. ${ }^{19}$ We first performed univariate analysis to select statistically significant risk factors. We then combined these risk factors with clinical considerations to conduct multivariate analysis, with adjustment for potential covariates, such as age and disease duration. Statistical significance for each variable in the model will be analysed by the Wald test.

All $\mathrm{p}$ values are two-sided, with the significance level set at 0.1 for univariate analysis and 0.05 for other statistical tests. To improve translation and dissemination of the findings in this project into real clinical practice, the final study findings will be analysed and reported according to the Strengthening the Reporting of Observational Studies in Epidemiology standards proposed by von Elm et al..$^{35}$ 


\section{Sample size estimation}

This study was primarily designed to test the change in CIMT in 5 years of follow-up compared with baseline in patients with SLE. The sample size was calculated using PASS software V.11.0. The parameter assumption was based on results from previous studies that an increase in CIMT in patients with SLE is $\sim 0.05-0.10 \mathrm{~mm}$ during 5 years. ${ }^{14}{ }^{15} \mathrm{We}$ conservatively assumed that the mean progression in CIMT was $0.05 \mathrm{~mm}$. We calculated that, with a SD of $0.20 \mathrm{~mm}$, a type I error probability of $5 \%$ and a power of $90 \%, 171$ participants will be required. Additionally, in considering the sample size to perform a linear mixed effect model, we calculated that, with a power of $>90 \%$ to detect a $10 \%$ difference in CIMT between subgroups of independent variables, 400 participants will be required. Allowing for a $10 \%$ dropout rate, we estimated that the final sample size will be $\sim 440$ patients.

\section{Patient and public involvement}

Although participants were not directly involved in the design of the study, we consulted patients and their families about the feasibility of the investigation. Results will be disseminated to the participants through newsletters in the outpatient department or patient meeting.

\section{Study status}

The baseline stages of the study have been completed. Follow-up started on October 2018 and is anticipated to be completed by December 2021. A paper reporting the main results of the whole project will then be submitted for publication.

\section{Ethics and dissemination}

Informed consent was obtained from all participants according to the Declaration of Helsinki on Biomedical Research Involving Human Studies.

The results from the main study regarding the primary objectives and findings will be submitted to peer-reviewed journals, as well as presented at national and international conferences. All data will be managed confidentially according to guidelines and legislation. We will apply for a patent for the technical details of construction of the database.

\section{DISCUSSION}

The current study has some unique features, which are different from previous studies ${ }^{13-15171824}$ First, to the best of our knowledge, most related previous studies were from Europe or North America, they comprised mostly Caucasian people, and some of the previous evidence was secondary analysis of data from placebo-controlled clinical trials with a short follow-up time. This study is the first prospective cohort to monitor CIMT in Chinese patients with SLE. Second, the sample size of this study is relatively large. This study will construct a Chinese population risk factor model and provide a robust parameter estimation about the progress of arteriosclerosis in patients with SLE in China. Furthermore, the data analysis attempts to compensate for individual factors that may affect progression of atherosclerosis. Our results may also help future development of guidelines and strategies for preventing arteriosclerosis in patients with SLE in China.

Subclinical atherosclerosis is an early finding and an important predictor of premature atherosclerosis and cardiovascular events in SLE. ${ }^{12}{ }^{36}$ To assess subclinical atherosclerosis, we used non-invasive imaging surrogate indices, such as CIMT as the primary outcome, which is the most widely used sensitive marker for the early stages of atherosclerosis, stroke and myocardial infarction. ${ }^{37-39}$ With regard to consensus definitions, the criteria used to measure CIMT are based on the published consensus statement recommendations regarding sonographic criteria.

Establishing long-term preoperative trajectories is the most challenging aspect of any SLE cohort study. Most patients with SLE in PUMCH should return for an outpatient review every 3-6 months. Regular periodic review of patients will ensure follow-up data collection in the current hospital-based prospective study.

This study has the following challenges. First, this is not an inception cohort. A pilot study showed that the median disease duration was 1.91 years at baseline, and $\sim 40 \%$ of patients were in their first year since SLE was diagnosed. A stratified disease duration will be considered in future sensitivity analysis. Second, all participants in this cohort study were recruited from a single centre of PUMCH. In this centre, ultrasound and laboratory testing are carried out in accordance with unified standards, and patients have good outpatient compliance of follow-up. Third, we did not enrol a control group. Progression of arteriosclerosis should be compared with baseline individually. Finally, most participants are from the north of China because of the geographical position of the hospital, and our results might only be representative of North China.

Despite these limitations, this protocol describes an approach to enable further testing of evidence concerning progression of arteriosclerosis in patients with SLE. We aim to identify clinically significant risk factors that can potentially guide management of patients with SLE. These results are expected to add further potential risk factors of SLE besides already known risk factors.

\section{Author affiliations}

${ }^{1}$ Medical Research Center, Peking Union Medical College Hospital, Chinese Academy of Medical Sciences \& Peking Union Medical College, Beijing, China ${ }^{2}$ Department of Cardiology, Peking Union Medical College Hospital, Chinese Academy of Medical Sciences \& Peking Union Medical College, Beijing, China ${ }^{3}$ Department of Rheumatology and Immunology, Peking Union Medical College Hospital, Chinese Academy of Medical Sciences \& Peking Union Medical College, Beijing, China

${ }^{4}$ Department of Gerontology, Peking Union Medical College Hospital, Chinese Academy of Medical Sciences \& Peking Union Medical College, Beijing, China

Acknowledgements We thank all patients who participated in the study. We thank Ellen Knapp, PhD, for editing the English text of a draft of this manuscript.

Contributors SZ, XZ, HP and YY conceived and planned the study protocol. HP, FD, $\mathrm{XY}, \mathrm{XY}, \mathrm{ML}, \mathrm{QW}, \mathrm{DX}, \mathrm{YF}$ and LK designed the questionnaire and are involved with data collection. HP was the main author involved in writing the manuscript and is involved with data analysis. All authors edited and approved the final manuscript. 
Funding This work was supported by the Beijing Municipal Science \& Technology Commission (Z131107002213005), the National Natural Science Foundation of China (grant number: 81641126), the Fundamental Research Funds for Central Universities (grant number: 2016RC330001) and the Chinese National Key Technology R\&D Program of the Ministry of Science and Technology (2008BAI59B02).

Competing interests None declared.

Patient consent for publication Obtained

Provenance and peer review Not commissioned; externally peer reviewed.

Open access This is an open access article distributed in accordance with the Creative Commons Attribution Non Commercial (CC BY-NC 4.0) license, which permits others to distribute, remix, adapt, build upon this work non-commercially, and license their derivative works on different terms, provided the original work is properly cited, appropriate credit is given, any changes made indicated, and the use is non-commercial. See: http://creativecommons.org/licenses/by-nc/4.0/.

\section{REFERENCES}

1. Liu Y, Kaplan MJ. Cardiovascular disease in systemic lupus erythematosus: an update. Curr Opin Rheumatol 2018;30:1-8.

2. Mok CC, Kwok CL, Ho LY, et al. Life expectancy, standardized mortality ratios, and causes of death in six rheumatic diseases in Hong Kong, China. Arthritis Rheum 2011;63:1182-9.

3. Ward MM. Premature morbidity from cardiovascular and cerebrovascular diseases in women with systemic lupus erythematosus. Arthritis Rheum 1999;42:338-46.

4. Bengtsson C, Öhman M-L, Nived O, et al. Cardiovascular event in systemic lupus erythematosus in northern Sweden: incidence and predictors in a 7-year follow-up study. Lupus 2012;21:452-9.

5. Libby P. Inflammation in atherosclerosis. Nature 2002;420:868-74.

6. Prechl J, Czirják L. The endothelial deprotection hypothesis for lupus pathogenesis: the dual role of $\mathrm{C} 1 \mathrm{q}$ as a mediator of clearance and regulator of endothelial permeability. F1000Res 2015;4.

7. Harris EN, Pierangeli SS, Primary PSS. Primary, secondary, and catastrophic antiphospholipid syndrome: what's in a name? Semin Thromb Hemost 2008;34:219-26.

8. Meroni PL, Raschi E, Testoni C, et al. Endothelial cell activation by antiphospholipid antibodies. Clin Immunol 2004;112:169-74.

9. Toloza SMA, Uribe AG, McGwin G, et al. Systemic lupus erythematosus in a multiethnic US cohort (LUMINA). XXIII. baseline predictors of vascular events. Arthritis Rheum 2004;50:3947-57.

10. Esdaile JM, Abrahamowicz M, Grodzicky T, et al. Traditional Framingham risk factors fail to fully account for accelerated atherosclerosis in systemic lupus erythematosus. Arthritis Rheum 2001;44:2331-7.

11. Schoenfeld SR, Kasturi S, Costenbader KH. The epidemiology of atherosclerotic cardiovascular disease among patients with SLE: a systematic review. Semin Arthritis Rheum 2013;43:77-95.

12. Roman MJ, Shanker B-A, Davis A, et al. Prevalence and correlates of accelerated atherosclerosis in systemic lupus erythematosus. $N$ Engl J Med 2003;349:2399-406.

13. Kiani AN, Post WS, Magder LS, et al. Predictors of progression in atherosclerosis over 2 years in systemic lupus erythematosus. Rheumatology 2011;50:2071-9.

14. Thompson T, Sutton-Tyrrell K, Wildman RP, et al. Progression of carotid intima-media thickness and plaque in women with systemic lupus erythematosus. Arthritis Rheum 2008;58:835-42.

15. Haque S, Skeoch S, Rakieh C, et al. Progression of subclinical and clinical cardiovascular disease in a UK SLE cohort: the role of classic and SLE-related factors. Lupus Sci Med 2018;5:e000267.

16. Jorge A, Lertratanakul A, Lee J, et al. Depression and progression of subclinical cardiovascular disease in systemic lupus erythematosus. Arthritis Care Res 2017;69:5-11.

17. Fadda S, Nassar H, Gamal SM, et al. Subclinical atherosclerosis in systemic lupus erythematosus patients and its relationship to disease activity and damage indices. Z Rheumatol 2015;74:529-32.
18. Lertratanakul A, Wu P, Dyer AR, et al. Risk factors in the progression of subclinical atherosclerosis in women with systemic lupus erythematosus. Arthritis Care Res 2014;66:1177-85.

19. Watad A, Abu Much A, Bracco D, et al. Association between ischemic heart disease and systemic lupus erythematosus-a large case-control study. Immunol Res 2017;65:459-63.

20. Fernández-Nebro A, Rúa-Figueroa Îñigo, López-Longo FJ, et al. Cardiovascular events in systemic lupus erythematosus: a nationwide study in Spain from the RELESSER registry. Medicine 2015;94:e1183.

21. Salmon JE, Roman MJ. Subclinical atherosclerosis in rheumatoid arthritis and systemic lupus erythematosus. Am J Med 2008;121:S3-S8.

22. Aviña-Zubieta JA, To F, Vostretsova K, et al. Risk of myocardial infarction and stroke in newly diagnosed systemic lupus erythematosus: a general population-based study. Arthritis Care Res 2017;69:849-56.

23. Hak AE, Karlson EW, Feskanich D, et al. Systemic lupus erythematosus and the risk of cardiovascular disease: results from the nurses' health study. Arthritis Rheum 2009;61:1396-402.

24. Wu G-C, Liu H-R, Leng R-X, et al. Subclinical atherosclerosis in patients with systemic lupus erythematosus: a systemic review and meta-analysis. Autoimmun Rev 2016;15:22-37.

25. Henrot P, Foret J, Barnetche T, et al. Assessment of subclinical atherosclerosis in systemic lupus erythematosus: a systematic review and meta-analysis. Joint Bone Spine 2018;85:155-63.

26. Li M, Zhang W, Leng X, et al. Chinese SLE treatment and research Group (CSTAR) registry: I. major clinical characteristics of Chinese patients with systemic lupus erythematosus. Lupus 2013;22:1192-9.

27. Li M, Wang Q, Zhao J, et al. Chinese SLE treatment and research Group (CSTAR) registry: II. prevalence and risk factors of pulmonary arterial hypertension in Chinese patients with systemic lupus erythematosus. Lupus 2014;23:1085-91.

28. Li J, Leng X, Li Z, et al. Chinese SLE treatment and research Group registry: III. Association of autoantibodies with clinical manifestations in Chinese patients with systemic lupus erythematosus. J Immunol Res 2014;2014:1-8.

29. Zhang S, Su J, Li X, et al. Chinese SLE treatment and research Group (CSTAR) registry: V. gender impact on Chinese patients with systemic lupus erythematosus. Lupus 2015;24:1267-75.

30. Zhao J, Bai W, Zhu P, et al. Chinese SLE treatment and research Group (CSTAR) registry VII: prevalence and clinical significance of serositis in Chinese patients with systemic lupus erythematosus. Lupus 2016;25:652-7.

31. Cheng Y, Li M, Zhao J, et al. Chinese SLE Treatment and Research Group (CSTAR) registry:VIII. Influence of socioeconomic and geographical variables on disease phenotype and activity in Chinese patients with SLE. Int J Rheum Dis 2018;21:716-24.

32. Hochberg MC. Updating the American College of rheumatology revised criteria for the classification of systemic lupus erythematosus. Arthritis Rheum $19971725 ; 40$.

33. Doria A, Shoenfeld Y, Wu R, et al. Risk factors for subclinical atherosclerosis in a prospective cohort of patients with systemic lupus erythematosus. Ann Rheum Dis 2003;62:1071-7.

34. Howard G, Sharrett AR, Heiss G, et al. Carotid artery intimal-medial thickness distribution in general populations as evaluated by B-mode ultrasound. ARIC Investigators. Stroke 1993;24:1297-304.

35. von Elm E, Altman DG, Egger M, et al. The strengthening the reporting of observational studies in epidemiology (STROBE) statement: guidelines for reporting observational studies. J Clin Epidemiol 2008;61:344-9.

36. Belibou C, Ancuta C, Ancuta E, et al. Carotid intima-media thickness and plaque as surrogate biomarkers of atherosclerosis among consecutive women with systemic lupus erythematosus. Rom J Morphol Embryol 2012;53:29-34.

37. O'Leary DH, Bots ML. Imaging of atherosclerosis: carotid intimamedia thickness. Eur Heart J 2010;31:1682-9.

38. Lorenz MW, Markus HS, Bots ML, et al. Prediction of clinical cardiovascular events with carotid intima-media thickness: a systematic review and meta-analysis. Circulation 2007;115:459-67.

39. Manzi S, Selzer F, Sutton-Tyrrell K, et al. Prevalence and risk factors of carotid plaque in women with systemic lupus erythematosus. Arthritis \& Rheumatism 1999;42:51-60. 